\title{
VAC.I4 - Plant-produced YFE-IT subunit vaccine against Yellow Fever induces humoral response in captive golden-headed lion tamarin (Leontopithecus chrysomelas)
}

André Tavares da Silva Fernandes ${ }^{1 \star}$; Luciane Pinto Gasparr; Renata Carvalho Pereira ${ }^{1}$; Vanessa de Oliveira Santos ${ }^{1}$; Renato Marchevsky ${ }^{1}$; Silvia Bahadian Moreira ${ }^{2}$; Alcides Pissinatti²; Elena Caride $^{1}$; Rosane Cuber Guimarães ${ }^{1}$; Marcos da Silva Freire ${ }^{1}$.

1Fiocruz/Bio-Manguinhos;

2Centro de Primatologia do Rio de Janeiro (CPRJ/INEA).

Introduction: Yellow Fever (YF) is a mosquito-borne viral hemorrhagic fever with a case fatality rate up to $50 \%$. It is endemic in the Brazilian Amazon region, and sporadic outbreaks take place outside the endemic area in Brazil. However, Brazil has recently experienced its largest recorded YF outbreak in decades, with 1,376 confirmed cases and 483 deaths in humans, and 864 confirmed epizooties in non-human primates (NHPs) from July 2017 to June 2018. Despite the excellent safety of YF 17DD vaccine, there are rare cases of serious adverse events, which are often fatal. According to described aspects, the risk of urban YF resurgence and the absence of a vaccine available for NHPs, there is a strong demand for the development of alternative YF vaccines that are safe and efficacious for disease control. Therefore, Bio-Manguinhos/Fiocruz, in collaboration with Fraunhofer USA Center for Molecular Biotechnology, has been developing a plant-derived recombinant subunit YF vaccine (YFE-1T) to address the global demand for increasing availability and safety of the YF vaccine. YFE-1T subunit composed by the ectodomain of the E protein was transiently expressed in Nicotiana benthamiana by Agrobacterium tumefaciens-mediated gene transfer, purified and adsorbed to aluminum hydroxide adjuvant.

Objective: In this study, the immunogenicity of the subunit YF vaccine in golden-headed lion tamarin (Leontopithecus chrysomelas) was evaluated by the determination of the neutralizing antibody titers $\left(\mathrm{PRNT}_{50}\right)$.

Methodology: NHPs were immunized by intramuscular route with two doses of YFE-1T with aluminum hydroxide adjuvant. All animal experiments were approved by the Animal Care and Use Committee (CEUA-UNIFESO 470/2018) and environmental license was authorized by the Brazilian Ministry of the Environment (ICMBio-SISBIO 60511-2/2018).

Results: YF neutralizing antibody titers were present after second dose in three of the six animals receiving YFE-1T with aluminum hydroxide. In this group, 50\% individuals seroconverted and one animal had high neutralizing titers. The control groups immunized with different doses of the live attenuated vaccine (groups 1, 2 and 3) showed 100\%, 40\% and $83.3 \%$ of seroconversion ninety days after inoculation, respectively.These findings suggest that new studies using other antigen concentrations and adjuvants are needed to produce a robust immunogenicity.

Conclusion: The results indicate the potential of this recombinant antigen for use in the development of a non-infectious YF vaccine. To evaluate the protective efficacy induced by subunit vaccine, one year after the first immunization all animals will be challenged using attenuated YF 17DD vaccine.

Keywords: Yellow Fever; subunit vaccine; preclinical study 